\title{
Determinants of Adolescent Physical Activity and Inactivity Patterns
}

\author{
Penny Gordon-Larsen, PhD*; Robert G. McMurray, PhD ; and Barry M. Popkin, PhD*§
}

\begin{abstract}
Objectives. Despite recognition of the important influence of environmental determinants on physical activity patterns, minimal empirical research has been done to assess the impact of environmental/ contextual determinants of physical activity. This article aims to investigate environmental and sociodemographic determinants of physical activity and inactivity patterns among subpopulations of US adolescents. We define environmental determinants as modifiable factors in the physical environment that impose a direct influence on the opportunity to engage in physical activity. The present research examines environmental and sociodemographic determinants of physical activity and inactivity with the implication that these findings can point toward societal-level intervention strategies for increasing physical activity and decreasing inactivity among adolescents.
\end{abstract}

Study Design and Methodology. The study population consists of nationally representative data from the 1996 National Longitudinal Study of Adolescent Health on 17766 US adolescents enrolled in US middle and high schools (including 3933 non-Hispanic blacks, 3148 Hispanics, and 1337 Asians). Hours/week of inactivity (TV/ video viewing and video/computer games) and times/ week of moderate to vigorous physical activity were collected by questionnaire. Outcome variables were moderate to vigorous physical activity and inactivity, which were broken into categories (physical activity: 0-2 times/week, 3-4 times/week, and $\geq 5$ times/week; inactivity: 0-10 hours/week, 11-24 hours/week, and $\geq 25$ hours/ week). Sociodemographic and environmental correlates of physical activity and inactivity were used as exposure and control variables and included sex, age, urban residence, participation in school physical education program, use of community recreation center, total reported incidents of serious crime in neighborhood, socioeconomic status, ethnicity, generation of residence in the United States, presence of mother/father in household, pregnancy status, work status, in-school status, region, and month of interview.

Logistic regression models of high versus low and medium physical activity and inactivity were used to investigate sex and ethnic interactions in relation to environmental and sociodemographic factors to examine evidence for the potential impact of physical education and recreation programs and sociodemographic factors on physical activity and inactivity patterns.

Results. Moderate to vigorous physical activity was lower and inactivity higher for non-Hispanic black and

From the *Carolina Population Center, ‡Department of Exercise Physiology, and §Department of Nutrition, School of Public Health, University of North Carolina, Chapel Hill, North Carolina.

Received for publication Sep 14, 1999; accepted Jan 12, 2000.

Reprint requests to (P.G.-L.) University of North Carolina at Chapel Hill, Carolina Population Center, CB 8120 University Square, 123 W Franklin St, Chapel Hill, NC 27516-3997. E-mail: gordon_larsen@unc.edu

PEDIATRICS (ISSN 0031 4005). Copyright $\odot 2000$ by the American Academy of Pediatrics.
Hispanic adolescents. Participation in school physical education programs was considerably low for these adolescents and decreased with age. Participation in daily school physical education (PE) program classes (adjusted odds ratio [AOR]: 2.21; confidence interval [CI]: 1.822.68) and use of a community recreation center (AOR: 1.75; CI: 1.56-1.96) were associated with an increased likelihood of engaging in high level moderate to vigorous physical activity. Maternal education was inversely associated with high inactivity patterns; for example, having a mother with a graduate or professional degree was associated with an AOR of .61 (CI: .48-.76) for high inactivity. High family income was associated with increased moderate to vigorous physical activity (AOR: 1.43; CI: 1.22-1.67) and decreased inactivity (AOR: .70; CI: .59-.82). High neighborhood serious crime level was associated with a decreased likelihood of falling in the highest category of moderate to vigorous physical activity (AOR: .77; CI: .66-.91).

Conclusions. These results show important associations between modifiable environmental factors, such as participation in school PE and community recreation programs, with activity patterns of adolescents. Despite the marked and significant impact of participation in school PE programs on physical activity patterns of US adolescents, few adolescents participated in such school PE programs; only $21.3 \%$ of all adolescents participated in 1 or more days per week of PE in their schools. In addition to the more readily modifiable factors, high crime level was significantly associated with a decrease in weekly moderate to vigorous physical activity.

The key modifiable factors that had an impact on physical activity did not affect inactivity. Thus, it is clear that physical activity and inactivity were associated with very different determinants. Although physical activity was most associated with environmental factors, inactivity was most associated with sociodemographic factors.

The data presented here confirm what researchers and pediatricians have known intuitively; however, these relationships have not been tested empirically, nor have they been studied in any nationally representative survey of US school-aged children. These findings show that patterns in inactivity cannot be explained using the environmental factors studied here and, thus, it is clearly important that researchers search for other environmental determinants likely to impact inactivity.

National-level strategies must include attention to school PE and community recreation programs, particularly for segments of the US population without access to resources and opportunities that allow participation in physical activity. Research to measure and explore the effects of other environmental determinants of activity and to ascertain whether there are any environmental determinants of inactivity are important future research directions. Pediatrics 2000;105(6). URL: http://www. pediatrics.org/cgi/content/full/105/6/e83; physical educa- 
tion, community recreation center, US adolescents, crime, socioeconomic factors, National Longitudinal Study of Adolescent Health.

ABBREVIATIONS. PE, physical education; Add Health, National Longitudinal Study of Adolescent Health; MET, metabolic equivalent; AOR, adjusted odds ratio; CI, confidence interval.

A dolescent overweight is a major US public health problem, with prevalence rates increasing for children and adolescents, ${ }^{1}$ particularly for minority ethnic groups. ${ }^{1,2}$ Minority adolescents have consistently high levels of inactivity and low levels of physical activity; these trends are exaggerated for females. ${ }^{3-5}$

Inactivity and activity are important biological determinants of obesity and represent major avenues for treating and preventing obesity. ${ }^{6-8}$ Physical activity has been associated with a wide range of beneficial health outcomes in adults, including bone and cardiovascular health and reduction of selected cancers. ${ }^{9}$ Inactivity, in particular, TV viewing, has been associated with obesity in cross-sectional studies of children, adolescents, and adults. ${ }^{10}$ Physical activity habits, and, specifically, inactivity, track significantly from adolescence to young adulthood. ${ }^{11}$

The physical activity literature has examined environmental determinants such as school and community sports and home access to fitness equipment, ${ }^{12,13}$ perceived physical environments, ${ }^{14}$ outdoor play spaces, ${ }^{15}$ time spent outdoors, ${ }^{16,17}$ exercise opportunity, 18 and "an environment that promotes excessive food intake and discourages physical activity." 19 A review of this topic concludes that little attention has been given to ecological determinants (eg, availability of facilities for activity and physical safety) and broader sociocultural determinants, including the influence of ethnicity on activity independent of confounding sociocultural factors. ${ }^{20} \mathrm{Sev}-$ eral researchers have suggested that school physical education (PE) programs and community recreation facilities are needed; however, little empirical research has been done to determine the impact of such facilities and programs on physical activity and inactivity levels of American adolescents. ${ }^{21}$

Furthermore, a literature review suggests that researchers often focus on univariate (as opposed to multivariate) relationships between single determinants and physical activity, ${ }^{22}$ despite the fact that physical activity has been shown to be influenced by the interaction among several factors. ${ }^{21,23}$

In the present article, we define environmental determinants as modifiable factors in the physical environment that impose a direct influence on the opportunity to engage in physical activity. The present research examines environmental and sociodemographic determinants of physical activity and inactivity with the implication that these findings can point toward societal-level intervention strategies for increasing physical activity and decreasing inactivity among US adolescents.

\section{Survey Design}

The study population consists of over 20000 adolescents enrolled in the National Longitunital Study of Adolescent Health (Add Health), a longitudinal, nationally representative, schoolbased sample of adolescents in grades 7 through 12 (ages: 11-21 years) in the United States. The Add Health study included a core sample and additional subsamples of selected ethnic and other groupings collected following informed consent procedures established by the institutional review board of the University of North Carolina at Chapel Hill. We used the wave I sample (20 747 eligible adolescents measured between April 1995 and December 1995), excluding adolescents who used a walking aid device (eg, cane, crutches, and wheelchair) and Native Americans $(n=178)$ because of small sample size. Our final sample totaled 17766 for prevalence estimates and logistic regression analysis. The survey design and sampling frame have been described elsewhere. ${ }^{2,3}$

In-school and in-home surveys of adolescents provided the activity data and components of the determinants data. In-home surveys of parents provided income, education, and other key sociodemographic data. Community data were collected from many sources, including 1993 Uniform Crime Reports, US Federal Bureau of Investigation. Race and ethnicity were determined using data from a combination of all the surveys.

\section{Physical Activity/Inactivity}

Moderate to vigorous physical activity (5-8 metabolic equivalents [METs]) and inactivity were assessed using standard 7-day recall (times/week) questionnaire methodology relevant for epidemiologic studies.,5,24-26 The assessment employed an array of questions similar to those used and validated in many other smaller studies to categorize adolescents into high, medium, and low activity and inactivity patterns with reasonable reliability and validity. The Add Health adolescents were asked about the times/ week spent in various physical activities (eg, "During the past week, how many times did you go roller-blading, roller-skating, skate-boarding, or bicycling?"). Each activity grouping was assigned a MET value $\left(1 \mathrm{MET}=3.5 \mathrm{~mL} \mathrm{O}_{2} / \mathrm{kg}\right.$ body weight/minute or resting metabolic rate) based on the Compendium of Physical Activity ${ }^{27}$ developed for adults to categorize activity as low, moderate, or vigorous. It is realized that the energy cost of activities is $\sim 10 \%$ higher in children; however, at the present time no norms exist for children. Higher intensity activities, such as skating, cycling, dance, martial arts activities, and active sports, were assigned 5 to $8 \mathrm{METs}$ and are, thus, considered moderate to vigorous physical activity.

Physical inactivity was assessed using standard 7-day recall questionnaire methodology relevant for epidemiologic studies ${ }^{4,5}$ used to categorize adolescents into high, medium, and low inactivity patterns. The inactivity questions follow the same structure as those validated for physical activity. The quantification of inactivity has received far less attention than physical activity ${ }^{28}$ and there is little published in the literature regarding reliability and validity of inactivity. In addition to the standard TV-viewing question used in other studies (eg, number of hours of TV viewing per week), Add Health elicited information on the number of hours and minutes of video viewing and video/computer game use during the past week. The quantification of inactivity is more straightforward than that of physical activity, because there is no concern with intensity and most sedentary activities have the same relative energy cost. In this study, all 3 inactivity variables were assigned 1 MET each. A composite inactivity score was calculated using the number of hours and minutes that each adolescent spent engaged in TV and video viewing and playing video/computer games. This strategy ignores potential differences in METs for computer/video games versus TV and video viewing, as well as differences attributable to advertisements seen on TV but not in video viewing.

\section{Study Variables}

Outcome variables were moderate to vigorous physical activity and inactivity, which were broken into categories (physical activity: 0-2 times/week, 3-4 times/week, and $\geq 5$ times/week; inactivity: 0-10 hours/week, 11-24 hours/week, and $\geq 25$ hours/ week) for comparability to published data. Sociodemographic and environmental correlates of physical activity and inactivity were 
used as exposure and control variables. These correlates included gender, age (11-21 years), total household income (0-\$26 200, $\$ 26200-\$ 50000$, and $\geq \$ 50000)$, maternal education $(<$ high school, high school/GED, some college, and college/professional degree), ethnic group (non-Hispanic white, non-Hispanic black, Hispanic [Cuban, Puerto Rican, Central/South American, Mexican/Chicano, and other Hispanic], and Asian [Chinese, Filipino, and other Asians]; ethnicity/race categorized from self-reports of adolescents and their parents), ethnicity and sex interaction, generation of residence in United States (1st, 2nd, or 3rd generation), urban residence, total reported incidents of serious crime per $100000,(0-4796,4800-7139$, and 7170-16 855), use of neighborhood recreation center, number of days in an average week that respondents attend PE classes at school (none, 1-4 days/week, and 5 days/week), whether respondent works for pay, region (Northeast, South, West, and Midwest), and month of interview. Control variables included pregnancy status, whether respondent was in school at time of interview, mother in household, father in household, family income predicted ( $n=3401 ; 19.1 \%)$, maternal education predicted $(n=258 ; 1.5 \%)$. Data on family income and maternal education were compiled using adolescent reports when parent reports were missing. A predication equation was used to estimate family income and maternal education when both parent and adolescent information were missing. Major correlates of interest are shown in Table 1.

\section{Statistical Analysis}

Statistical analyses were conducted using STATA (STATA, Chicago, IL). ${ }^{29}$ Poststratification sample weights were used in all analyses to allow these results to be comparable with adolescents attending schools in the United States. Survey design effects of multiple stages of cluster sampling were also controlled for in all analyses. Logistic regression models of physical activity and inactivity were used to investigate sex and ethnic interactions in relation to environmental and sociodemographic correlates of physical activity and inactivity to examine evidence for the potential impact of these correlates (eg, PE programs and community recreation facilities) on physical activity and inactivity patterns. Models compare high activity (or inactivity) versus low and me-

TABLE 1. Sample Characteristics*

\begin{tabular}{|c|c|c|}
\hline Correlate & Proportion & Number \\
\hline \multicolumn{3}{|l|}{ Sex } \\
\hline Male & $50.8 \%$ & 8727 \\
\hline Female & $49.2 \%$ & 9039 \\
\hline \multicolumn{3}{|l|}{ Age } \\
\hline Mean age & $15.5+-.12$ & $11-21 \mathrm{y}$ \\
\hline \multicolumn{3}{|l|}{ Ethnicity/race } \\
\hline Non-Hispanic white & $66.7 \%$ & 9348 \\
\hline Non-Hispanic black & $16.7 \%$ & 3933 \\
\hline Hispanic & $12.7 \%$ & 3148 \\
\hline Asian & $4.0 \%$ & 1337 \\
\hline \multicolumn{3}{|l|}{ Maternal education } \\
\hline$<$ High school & $16.2 \%$ & 2993 \\
\hline $\begin{array}{l}\text { High school/general education } \\
\text { development }\end{array}$ & $32.4 \%$ & 5297 \\
\hline Some college & $25.8 \%$ & 4512 \\
\hline College degree & $13.3 \%$ & 2623 \\
\hline Graduate/professional degree & $7.6 \%$ & 1496 \\
\hline \multicolumn{3}{|l|}{ Family income } \\
\hline Low $(\$ 0-\$ 26200)$ & $32.3 \%$ & 5798 \\
\hline Middle (\$26.2-\$50 000) & $37.0 \%$ & 6642 \\
\hline High $(\$ 50000+)$ & $30.6 \%$ & 5326 \\
\hline \multicolumn{3}{|l|}{ Recreation center use } \\
\hline Use center & $80.4 \%$ & 14083 \\
\hline Don't use center & $19.6 \%$ & 3683 \\
\hline \multicolumn{3}{|l|}{ Weekly PE } \\
\hline 0 times/wk & $78.7 \%$ & 13962 \\
\hline 1-4 times/wk & $6.7 \%$ & 1276 \\
\hline 5 times/wk & $14.6 \%$ & 2528 \\
\hline \multicolumn{3}{|l|}{ Crime level (total crime/100 000) } \\
\hline Low (0-4 796) & $37.4 \%$ & 6189 \\
\hline Medium (4800-7139) & $33.2 \%$ & 6503 \\
\hline High (7170-16 855) & $29.4 \%$ & 5074 \\
\hline
\end{tabular}

* Figures are weighted to be nationally representative. dium activity (or inactivity), with non-Hispanic whites as the reference category. Regression models were used to investigate the association of environmental correlates of physical activity with age, sex, and ethnicity.

\section{RESULTS}

\section{Ethnicity and Gender}

Table 2 presents the proportion of adolescents who participated in low, moderate, and high categories of physical activity. Among males, the proportion of adolescents to participate in the highest category of moderate to vigorous physical activity varied little by ethnicity. In contrast, among females, a greater percentage of non-Hispanic whites and Asians participated in the highest category moderate to vigorous physical activity, whereas the proportion was smaller for non-Hispanic blacks and Hispanics.

Results for physical inactivity show greater ethnic variability than for activity. Table 2 presents the proportion of adolescents to participate in low, medium, and high categories of weekly inactivity (TV/video viewing and video/computer game use). The proportion of adolescents to participate in the highest category of inactivity was greatest for non-Hispanic black males and females and Hispanic males and females and was lowest for Asian and non-Hispanic white females.

\section{Environmental Determinants of Physical Activity}

Table 3 shows the distribution of sociodemographic and environmental determinants of physical activity and inactivity by ethnicity. Most adolescents were not enrolled in PE courses at the time of survey $(78.7 \%)$ and only a fraction $(14.6 \%)$ had PE classes on 5 or more days. Results varied by ethnicity; fewer non-Hispanic whites had PE classes and a greater proportion of Asians had PE. A small percentage of adolescents used a neighborhood community recreation center $(19.6 \%)$ with the greatest percentage seen for non-Hispanic blacks (23.6\%). Results for PE and recreation center use did vary by sex; more males were enrolled in PE classes and used community recreation centers than females. Results show that the highest percentage of non-Hispanic whites $(46.9 \%)$ lived in low crime areas, whereas the highest percentage of non-Hispanic blacks (58.1\%) and Hispanics $(41.5 \%)$ lived in high crime areas. Maternal education was lowest for Hispanics $(44.7 \%$ had less than a high school degree) and highest for Asians $(11.1 \%)$ and non-Hispanic whites $(8.5 \%)$ with given percentages of mothers with graduate or professional degrees. The greatest percentage of nonHispanic whites (38.1\%) and Asians (34.8\%) had high family incomes, whereas the greatest percentage of non-Hispanic black (58\%) and Hispanic $(52.5 \%)$ had low family incomes.

\section{School PE}

Participation in school PE programs was substantially associated with likelihood of engaging in moderate to vigorous physical activity (Table 4). Having PE 1 to 4 times per week was associated with an increase of $44 \%$ in falling in the highest category of moderate to vigorous physical activity (adjusted 
TABLE 2. Weighted Proportion of Adolescents Participating in Given Categories of Physical Activity and Inactivity*

\begin{tabular}{|c|c|c|c|c|c|c|c|c|}
\hline \multirow[t]{2}{*}{ Activity Level } & \multicolumn{2}{|c|}{$\begin{array}{c}\text { Non-Hispanic } \\
\text { Whites }\end{array}$} & \multicolumn{2}{|c|}{$\begin{array}{c}\text { Non-Hispanic } \\
\text { Blacks }\end{array}$} & \multicolumn{2}{|c|}{ Hispanics } & \multicolumn{2}{|c|}{ Asians } \\
\hline & Males & Females & Males & Females & Males & Females & Males & Females \\
\hline Low & 26.5 & 37.3 & 24.9 & 46.8 & 24.9 & 42.6 & 21.1 & 37.6 \\
\hline Medium & 30.1 & 34.2 & 34.1 & 34.3 & 31.6 & 35.9 & 31.0 & 34.3 \\
\hline High & 43.4 & 28.5 & 41.0 & 18.9 & 43.4 & 21.4 & 47.9 & 28.0 \\
\hline Medium & 34.9 & 35.2 & 26.4 & 26.8 & 32.9 & 29.7 & 36.9 & 30.9 \\
\hline High & 35.6 & 22.8 & 51.6 & 44.7 & 39.4 & 34.0 & 35.9 & 28.9 \\
\hline
\end{tabular}

* Results are weighted to be nationally representative and standard error terms are adjusted for complex survey design effects.

TABLE 3. Sociodemographic and Environmental Determinants of Physical Activity and Inactivity*

\begin{tabular}{|c|c|c|c|c|}
\hline Variable & $\begin{array}{c}\text { Non-Hispanic Whites } \\
n=9348 \\
\%(\mathrm{SE})\end{array}$ & $\begin{array}{c}\text { Non-Hispanic Blacks } \\
n=3933 \\
\%(\mathrm{SE})\end{array}$ & $\begin{array}{l}\text { Hispanic } \\
n=3148 \\
\%(\mathrm{SE})\end{array}$ & $\begin{array}{c}\text { Asian } \\
n=1337 \\
\%(\mathrm{SE})\end{array}$ \\
\hline \multicolumn{5}{|l|}{ PE d/wk } \\
\hline $0 \mathrm{~d} / \mathrm{wk}$ & $80.9(1.4)$ & $75.2(3.1)$ & $74.5(2.4)$ & $70.6(3.1)$ \\
\hline $1-4 \mathrm{~d} / \mathrm{wk}$ & $6.4(1.1)$ & $6.5(1.4)$ & $7.8(1.7)$ & $8.6(3.4)$ \\
\hline $5 \mathrm{~d} / \mathrm{wk}$ & $12.6(1.2)$ & $18.3(2.9)$ & $17.7(2.2)$ & $20.8(4.0)$ \\
\hline \multicolumn{5}{|l|}{ Recreation center use } \\
\hline Use center & $18.9(1.3)$ & $23.6(2.1)$ & $18.5(1.0)$ & $18.2(2.2)$ \\
\hline Don't use center & $81.1(1.3)$ & $76.4(2.1)$ & $81.5(1.0)$ & $81.8(2.2)$ \\
\hline \multicolumn{5}{|l|}{ Total incidents serious } \\
\hline \multicolumn{5}{|l|}{ Crime/100 000} \\
\hline $0-4795$ & $46.9(5.7)$ & $14.3(4.0)$ & $19.7(5.7)$ & $32.0(11.7)$ \\
\hline $4800-7139$ & $32.7(5.0)$ & $27.5(6.7)$ & $38.8(8.1)$ & $47.2(11.8)$ \\
\hline $7170-16855$ & $20.4(4.1)$ & $58.1(7.3)$ & $41.5(8.5)$ & $20.8(6.7)$ \\
\hline \multicolumn{5}{|l|}{ Maternal education } \\
\hline Less than high school & $10.1(.9)$ & $19.0(2.1)$ & $44.7(3.5)$ & $20.6(4.2)$ \\
\hline High school/general education development & $34.1(1.3)$ & $35.4(1.8)$ & $23.1(1.9)$ & $20.6(2.0)$ \\
\hline Some college & $28.2(.9)$ & $24.5(1.5)$ & $17.7(1.3)$ & $16.6(2.6)$ \\
\hline College degree & $14.7(.9)$ & $10.0(.9)$ & $6.2(.8)$ & $26.6(3.1)$ \\
\hline Graduate professional degree & $8.5(.9)$ & $6.4(1.3)$ & $3.5(.8)$ & $11.1(1.7)$ \\
\hline \multicolumn{5}{|l|}{ Family income } \\
\hline$\$ 0-\$ 26200$ & $22.6(1.8)$ & $58.0(2.9)$ & 52.5 & $24.5(3.1)$ \\
\hline$\$ 26200-\$ 50000$ & $39.3(1.1)$ & $29.3(1.8)$ & $33.8(1.9)$ & $40.7(3.2)$ \\
\hline$\$ 50000$ & $38.1(2.2)$ & $12.7(1.6)$ & $13.7(1.8)$ & $34.8(3.0)$ \\
\hline
\end{tabular}

SE indicates standard error.

* Results are weighted to be nationally representative and standard error terms are adjusted for complex survey design effects.

odds ratio [AOR]: 1.44; 95\% confidence interval [CI]: 1.09-1.92; $P \leq .01$ ) and having PE 5 times per week was associated with a substantial increase in likelihood of falling in the highest category of moderatevigorous physical activity (AOR: 2.21; CI: 1.82-2.68; $P \leq .00001)$. Participation in PE was not significantly associated with likelihood of engaging in high levels of inactivity (Table 4).

We found marked differences in the patterns of PE program participation by age and gender (Fig 1). For example, 12-year-olds $(24.7 \%)$ had a higher frequency of $\mathrm{PE}$, but this number decreased with age, so that by 17 years of age, only $8.3 \%$ of adolescents had PE 1 or more times per week. Males (15.3\%) were more likely than females (13.7\%) to participate in PE at least once per week. Regression analyses modeling use of PE programs, using ethnicity, sex, age, and a sex-ethnicity interaction, show that age $(P \leq .00001)$; sex $(P \leq .012)$; and Non-Hispanic black $(P \leq .010)$, Asian $(P \leq .005)$, and Hispanic $(P \leq .014)$ ethnicity (but not sex-ethnicity interactions) were important factors in PE use.
Neighborhood Community Recreation Centers

Use of a community recreation center also had a marked association with likelihood of engaging in moderate to vigorous physical activity (Table 4). Using a recreation center was associated with a $75 \%$ increase in the likelihood of falling in the highest category of moderate to vigorous physical activity (AOR: $1.75 ; \mathrm{CI}: 1.56-1.96 ; P \leq .00001)$. As with school $\mathrm{PE}$, inactivity did not vary with use of a community recreation center (Table 4). Recreation center use was modeled similarly to that described above for PE use. Results show that sex $(P \leq .021)$, non-Hispanic black ethnicity $(P \leq .006)$ and its sex interaction $(P \leq .005)$, and the interaction between Hispanic ethnicity and sex $(P \leq .018)$ were important factors in recreation center use.

\section{Total Reported Incidents of Serious Crime}

High crime levels were associated with a decrease in the likelihood of falling in the highest category of moderate to vigorous physical activity (AOR: .77; CI: $.66-.91 ; P \leq .002)$, although the magnitude of this association was not as great as that seen for school 
TABLE 4. AORs for Risk of High Levels of Physical Activity and Inactivity Among Given Environmental Contexts*十

\begin{tabular}{|c|c|c|c|}
\hline Context & AOR & $95 \% \mathrm{CI}$ & $P$ Value \\
\hline \multicolumn{4}{|c|}{ Highest category of moderate to vigorous physical activity } \\
\hline \multicolumn{4}{|c|}{ PE times/wk } \\
\hline 0 times/wk & 1.00 & - & - \\
\hline 1-4 times/wk & 1.44 & $1.09-1.92$ & .01 \\
\hline 5 times/wk & 2.21 & $1.82-2.68$ & .00001 \\
\hline \multicolumn{4}{|l|}{ Recreation center use } \\
\hline Don't use center & 1.00 & - & - \\
\hline Use center & 1.75 & $1.56-1.96$ & .00001 \\
\hline \multicolumn{4}{|l|}{ Total crime/100 000} \\
\hline Low & 1.00 & - & - \\
\hline Medium & .89 & $.78-1.02$ & NS \\
\hline High & .77 & $.66-.91$ & .002 \\
\hline \multicolumn{4}{|l|}{ Highest category of inactivity } \\
\hline \multicolumn{4}{|l|}{$\mathrm{PE}$ times/wk } \\
\hline 0 times/wk & 1.00 & - & - \\
\hline 1-4 times/wk & .92 & $.75-1.13$ & NS \\
\hline 5 times/wk & .96 & $.82-1.14$ & NS \\
\hline \multicolumn{4}{|l|}{ Recreation center use } \\
\hline Don't use center & 1.00 & - & - \\
\hline Use center & 1.01 & $.89-1.13$ & NS \\
\hline \multicolumn{4}{|l|}{ Total crime/100 000} \\
\hline Low & 1.00 & - & - \\
\hline Medium & 1.11 & $.94-1.32$ & NS \\
\hline High & 1.13 & $.98-1.60$ & NS \\
\hline
\end{tabular}

NS indicates not significant.

* Adjusted using logistic regression models controlling for sex, age, urban residence, socioeconomic status, ethnicity, generation of residence in the United States, presence of mother/father in household, pregnancy status, in-school status, work status, region, and month of interview.

+ Results are weighted to be nationally representative and standard error terms are adjusted for complex survey design effects.



Fig 1. Weighted percentage of US adolescents who participate in school PE programs. Weighted to be nationally representative with the error terms corrected for design effects.

PE or community recreation center use (Table 4). High crime was associated with an increase in the likelihood of engaging in high levels of inactivity; however, this association was not significant (Table 4). Again, crime was modeled similarly to that described above for PE use and was very clearly associated with non-Hispanic black $(P \leq .00001)$ and Hispanic $(P \leq .00001)$ ethnicity, but there were no significant age, sex, and sex-ethnicity interactions.

\section{Seasonality and Region}

Classic work on environmental determinants of physical activity has focused on season and region of residence. Our results do not show major effects of seasonality and region. For physical activity, there was no relationship with month during which activity patterns were measured, and, thus, no evidence of a seasonality effect. However, there was a significant association with residence in the Northeast re- gion (AOR: 1.27; CI: 1.05-1.56; $P \leq .015$ ). For composite inactivity, there was no region effect. There was a significant association with inactivity measured during July (AOR: 1.32; CI: 1.09-1.68; P $\leq .006$ ) with inactivity.

\section{Sociodemographic Determinants of Physical Activity} Maternal Education

High level of maternal education (adolescents with mothers who had graduate or professional degrees) was significantly associated with increased likelihood of having high levels of moderate to vigorous physical activity (AOR: 1.27; CI: 1.01-1.60; $P \leq .045$ ). At other levels, maternal education was not significantly associated with high category of moderate to vigorous physical activity, although likelihood of falling in the highest category of moderate to vigorous physical activity increased with maternal education (Table 5). Conversely, having a mother with some college education (AOR: .84; CI: .72-.99; $P \leq$ .044), a college degree (AOR: .80; CI: .66-.98; $P \leq$ $.028)$, or having a graduate or professional degree (AOR: .61; CI: .48-.76; $P \leq .00001$ ) was associated with decreased likelihood of engaging in high levels of inactivity (Table 5).

\section{Family Income}

Family income was associated with both physical activity and inactivity (Table 5). Adolescents from households with highest family income had an increased likelihood of falling in the highest category of moderate to vigorous physical activity (AOR: 1.43; CI: 1.22-1.67; $P \leq .00001)$ and decreased likelihood of falling in the highest category of inactivity (AOR: .70; CI: .59-.82; $P \leq$.00001). In addition, adolescents of 
TABLE 5. AORs for Risk of High Levels of Physical Activity and Inactivity Among Given Sociodemographic Contexts*†

\begin{tabular}{|c|c|c|c|}
\hline Context & AOR & $95 \% \mathrm{CI}$ & $P$ Value \\
\hline \multicolumn{4}{|l|}{$\begin{array}{l}\text { Highest category of moderate } \\
\text { to vigorous physical } \\
\text { activity }\end{array}$} \\
\hline \multicolumn{4}{|l|}{ Maternal education } \\
\hline Less than high school & 1.00 & - & - \\
\hline $\begin{array}{l}\text { High school/general } \\
\text { education development }\end{array}$ & .93 & $.79-1.12$ & NS \\
\hline Some college & 1.05 & $.88-1.25$ & NS \\
\hline College degree & 1.12 & $.90-1.38$ & NS \\
\hline $\begin{array}{l}\text { Graduate/professional } \\
\text { degree }\end{array}$ & 1.27 & $1.01-1.60$ & .045 \\
\hline \multicolumn{4}{|l|}{ Family income } \\
\hline Low & 1.00 & - & - \\
\hline Medium & 1.19 & $1.04-1.36$ & .013 \\
\hline High & 1.43 & $1.22-1.67$ & .00001 \\
\hline \multicolumn{4}{|l|}{ Highest category of inactivity } \\
\hline \multicolumn{4}{|l|}{ Maternal education } \\
\hline Less than high school & 1.00 & - & - \\
\hline $\begin{array}{l}\text { High school/general } \\
\text { education development }\end{array}$ & 1.00 & $.87-1.45$ & NS \\
\hline Some college & .84 & $.72-.99$ & .044 \\
\hline College degree & .80 & $.66-.98$ & .028 \\
\hline $\begin{array}{l}\text { Graduate/professional } \\
\text { degree }\end{array}$ & .61 & $.48-.76$ & .00001 \\
\hline \multicolumn{4}{|l|}{ Family income } \\
\hline Low & 1.00 & - & - \\
\hline Medium & .93 & $.82-1.05$ & NS \\
\hline High & .70 & $.59-0.82$ & .00001 \\
\hline
\end{tabular}

NS indicates not significant.

* Adjusted using logistic regression models controlling for sex, age, urban residence, socioeconomic status, ethnicity, presence of mother/father in household, pregnancy status, in-school status, work status, region, and month of interview.

t Results are weighted to be nationally representative and standard error terms are adjusted for complex survey design effects.

medium family income were at increased likelihood of falling in the highest category of moderate to vigorous physical activity (AOR: 1.19; CI: 1.04-1.36; $P \leq$.013).

\section{Interactions Between Ethnicity and Sex and Determinants}

Logistic regression models were used to test sex and ethnicity interactions with each set of environmental determinants (PE participation, recreation center use, and neighborhood crime). Differential effects were seen only for inactivity for neighborhood crime and sex and recreation center and race. Females living in high crime areas were at increased likelihood of falling in the highest category of inactivity (AOR: 1.29; CI: 1.03-1.62; $P \leq .027$ ). NonHispanic black ethnicity and recreation center use were associated with an increase in likelihood of inactivity (AOR: 1.61; CI: 1.22-2.11; $P \leq .001$ ).

\section{Effect of Determinants on Physical Activity and Inactivity Among Subpopulations}

The above analysis, repeated using the subpopulation groups of Hispanics (Cuban, Puerto Rican, Central/South American, Mexican/Chicano, and other Hispanics) and Asians (Chinese, Filipino, and other Asians) showed small and nonsignificant differences for physical activity. Adjusted odds ratios for inactivity were significant for Filipino (AOR: 1.71; CI: 1.05-2.80; $P \leq .032$ ), non-Hispanic black (AOR:
1.86; CI: $1.54-2.24 ; P \leq .0001)$, and Puerto Rican (AOR: 1.46; CI: 1.10-1.95; $P \leq .009$ ) adolescents.

\section{DISCUSSION}

Add Health, a unique survey with its rich sample of ethnic subpopulations and detailed activity and inactivity, sociodemographic, and environmental data, investigates modifiable physical activity determinants vitally important to current efforts to increase physical activity among our nation's adolescents. The high levels of obesity and inactivity and low levels of physical activity illustrate the public health importance of this analysis. ${ }^{3}$

The results fit well with US teen obesity patterns. ${ }^{2}$ Inactivity was highest and physical activity lowest for non-Hispanic black and Hispanic adolescents. These trends were exaggerated for females and older adolescents.

\section{PE Matters but Few Participate}

The national push away from comprehensive PE in US schools is remarkable. ${ }^{8}$ Although our results show important associations between participation in PE and activity patterns, particularly on a daily basis, few teens receive PE. Our results indicate that PE classes may represent the only opportunity for many adolescents to engage in weekly physical activity. Conversely, the number of PE classes per week was not associated with level of inactivity of these adolescents. As shown, age, sex, and ethnicity were important factors in PE use. Clearly, these are modifiable relationships. Indeed, the Child and Adolescent Trial for Cardiovascular Health has shown that a program aimed at improving school PE has been successful in increasing moderate to vigorous physical activity in PE classes. ${ }^{30}$

Logistic regression results show a strong impact of PE programs on physical activity patterns. To place these results in context of potential program outcome, simulations run from the logistic regression models (Tables 4 and 5) show increases in adjusted proportion of adolescents participating in the highest category of moderate to vigorous physical activity by number of days of PE per week. For example, the adjusted proportion of adolescents to fall in the highest category of moderate to vigorous physical activity increases from $30.3 \%$ for those adolescents with no weekly PE to $37.6 \%$ for those having PE 1 to 4 times per week and to $46.8 \%$ for those having PE 5 times per week. This represents a marked increase in the percentage of adolescents who would be participating in substantial levels of weekly moderate to vigorous physical activity.

\section{Community Recreation Facilities Might Offer a Key Intervention}

Adolescents who used a community recreation center (as with those in PE) reported markedly higher levels of moderate to vigorous physical activity than those who did not. As indicated, sex, nonHispanic black ethnicity (with a strong ethnicity-sex interaction) and Hispanic female ethnicity were important factors in community recreation center use. 


\section{Crime Reduces Physical Activity}

Total number of incidents of serious crime in the adolescents' neighborhood was significantly associated with a decrease in physical activity. Crime was also associated with an increase in inactivity, although this relationship was not significant. Crime was very clearly associated with ethnicity, but there were no significant age, sex, and sex-ethnicity interactions. However, logistic regression results showed a differential association between high neighborhood crime and increased inactivity (AOR: 1.29; CI: 1.031.62; $P \leq .027$ ) for females relative to males.

\section{Key Environmental Factors Do Not Reduce Inactivity}

The key modifiable factors that had an impact on activity did not affect inactivity. Thus, it is clear that physical activity and inactivity were influenced by very different determinants. Although physical activity was most influenced by environmental factors, inactivity was much more influenced by sociodemographic factors. Higher socioeconomic status measured by maternal education and family income had a substantial impact on likelihood of engaging in inactivity. Advanced education and high income were associated with lower levels of inactivity.

\section{Related Research}

Other scholars have found that participation in community sports is an important predictor of physical activity and have suggested the need for improving access to community-based physical activity opportunities, $^{13}$ health-oriented improvements in PE programs, ${ }^{31}$ daily PE programs that are fun and stress lifelong physical activity habits, ${ }^{19}$ and a possible role for clinicians in educating parents about appropriate community play places. ${ }^{15}$

This study has 1 limitation. The data on community recreation centers were based on actual use, not availability. Data on availability are unavailable in any national database. This actual use response may produce misleading results because physically active people may be more likely to use recreation centers. Additional research is needed to determine the types of facilities that might offset the impact of crime on physical activity and inactivity.

The data presented here confirm what researchers and pediatricians have known intuitively; however, these relationships have not been tested empirically, nor have they been studied in any national dataset. These findings show that patterns in inactivity cannot be explained using the environmental factors studied here and, thus, it is clearly important that researchers search for other environmental determinants likely to impact inactivity. This further demonstrates the difficulty that researchers and pediatricians face in developing public health efforts to decrease inactivity.

The results of this study show the importance of PE classes and recreation centers in increasing physical activity and give powerful evidence supporting the importance of increasing opportunities for physical activity and the potential impact of PE programs and community recreation programs on physical ac- tivity of US adolescents. In addition, it is imperative that we provide safe and accessible places for exercise for our nation's youth. In many communities, the only such place may be the school.

Clearly our national public health initiatives should consider these options. More research is needed on the role of these factors in affecting activity and inactivity and on ways to most effectively change them. PE and community recreation programs should receive attention at a national level, particularly for segments of the population without resources to locate and pay for extracurricular and extracommunity physical activity opportunities. Availability of such resources will increase success for pediatricians in recommendations to patients to increase physical activity and decrease inactivity. This research also suggests that with increased opportunities for physical activity, adolescents may opt to selectively engage in these activities instead of more inactive behaviors.

\section{ACKNOWLEDGMENTS}

This work was supported in part by National Institute of Child Health and Human Development Grant P01-HD31921 and the Dannon Institute Postdoctoral Fellowship in Interdisciplinary Nutrition Science.

We thank Frances Dancy for her helpful administrative assistance and Tom Swasey for assistance with the graphics.

\section{REFERENCES}

1. Troiano RP, Flegal KM, Kuczmarski RJ, Campbell SM, Johnson CL. Overweight prevalence and trends for children and adolescents. Arch Pediatr Adolesc Med. 1995;149:1085-1091

2. Popkin BM, Udry JR. Adolescent obesity increases significantly in second and third generation US Immigrants: the National Health and Longitudinal Study of Adolescent Health. J Nutr. 1998;128:701-706

3. Gordon-Larsen P, McMurray RG, Popkin BM. Adolescent physical activity and inactivity vary by ethnicity: the National Longitudinal Study of Adolescent Health. J Pediatr. 1999;135:301-306

4. Heath GW, Pratt M, Warren CW, Kann L. Physical activity patterns in American high school students: results from the 1990 Youth Risk Behavior Survey. Arch Pediatr Adolesc Med. 1994;148:1131-1136

5. Andersen RE, CJ Crespo, SJ Bartlett, LJ Cheskin, M Pratt. Relationship of physical activity and television watching with body weight and level of fatness among children: results from the Third National Health and Nutrition Examination Survey. JAMA. 1998;279:938-942

6. Epstein LH, Saelens BE, O'Brien JG. Effects of reinforcing increases in activity versus decreases in sedentary behavior in obese children. Int J Behav Med. 1995;2:41-50

7. Epstein LH, Saelens BE, Myers MD, Vito D. Effects of decreasing sedentary behaviors on activity choice in obese children. Health Psychol. 1997;16:107-113

8. US Department of Health and Human Services. Physical Activity and Health: A Report of the Surgeon General. Atlanta, GA: US Department of Health and Human Services, Centers for Disease Control and Prevention, National Center for Chronic Disease Prevention and Health Promotion; 1996

9. National Institutes of Health. Consensus development conference on diet and exercise in non-insulin dependent diabetes mellitus. Diabetes Care. 1987; 10:639-643

10. Gortmaker SL, Sobal AM, Peterson K, Colditz CA, Dietz WH. Television viewing as a cause of increasing obesity among children in the United States. Arch Pediatr Adolesc Med. 1996;150:536-562

11. Raitakari OT, Porkka KVK, Taimela S, et al. Effects of persistent physical activity and inactivity on coronary risk factors in children and young adults: the Cardiovascular Risk in Young Finns Study. Am J Epidemiol. 1994;140:195-205

12. Trost SG, Pate RR, Dowda M, et al. Gender differences in physical activity and determinants of physical activity in rural fifth grade children. J Sch Health. 1996;66:145-150

13. Trost SG, Pate RR, Saunders R, Ward DS, Dowda M, Felton G. A prospective study of determinants of physical activity in rural fifth 
grade children. Prev Med. 1997;26:257-263

14. Sallis JF, Johson MF, Calfas KJ, Caparosa S, Nichols JF. Assessing perceived physical environmental variables that may influence physical activity. Res Q Exerc Sport. 1997;68:345-351

15. Sallis JF, McKenzie TL, Elder JP, Broyles SL, Nader PR. Factors parents use in selecting play spaces for young children. Arch Pediatr Adolesc Med. 1997;151:414-417

16. Baranowski T, Thompson WO, DuRant RH, Baranowski J, Puhl J. Observations on physical activity in physical locations: age, gender, ethnicity, and month effects. Res Q Exerc Sport. 1993;64:127-133

17. Klesges RC, Eck LH, Hanson CL, Haddock CK, Klesges LM. Effects of obesity, social interactions, and physical environment on physical activity in preschoolers. Health Psychol. 1990;9:435-449

18. Bungum TJ, Vincent ML. Determinants of physical activity among female adolescents. Am J Prev Med. 1997;13:115-122

19. Hill JO, Peters JC. Environmental contributions to the obesity epidemic. Science. 1998;280:1371-1374

20. Goran MI, Reynolds KD, Lindquist CH. Role of physical activity in the prevention of obesity in children. Int J Obes Relat Metab Disord. 1999; 23(suppl 3):S18-S33

21. Sallis JF, Simons-Morton BG, Stone EJ, et al. Determinants of physical activity and interventions in youth. Med Sci Sport Exerc. 1992;24: S248-S257

22. Khol HW, Hobbs KE. Development of physical activity behaviors among children and adolescents. Pediatrics. 1998;101:549-554

23. Taylor WC, Sallis JF. Determinants of physical activity in children. World Rev Nutr Diet. 1997;82:159-167

24. Baranowski T. Validity and reliability of self-report measures of physical activity: an information processing perspective. Res Q Exerc Sport. 1988;59:314-327

25. Pate RR, Heath GW, Dowda M, Trost SG. Associations between physical activity and other health behaviors in a representative sample of US adolescents. Am J Public Health. 1996;86:1577-1581

26. Sallis JF, Buono MJ, Roby JJ, Micale FG, Nelson JA. Seven-day recall and other physical activity self-reports in children and adolescents. Med Sci Sport Exerc. 1993;25:99-108

27. Ainsworth B, Haskell WL, Leon AS, et al. Compendium of physical activities: Classification of energy costs of human physical activities. Med Sci Sport Exerc. 1993;25:71-80

28. Dietz WH. The role of lifestyle in health: the epidemiology and consequences of inactivity. Proc Nutr Soc. 1996;55:829-840

29. Stata Corporation. Stata Statistical Software, Release 6.0. College Station, TX: Stata Corporation; 1999

30. Luepker RV, Perry CL, McKinlay SM, et al. Outcomes of a field trial to improve children's dietary patterns and physical activity: the Child and Adolescent Trial for Cardiovascular Health. JAMA. 1996;275:768-776

31. Pate RR, Corbin CB, Simons-Morton BG, Ross JG. Physical education and its role in school health promotion. J Sch Health. 1987;57:445-450 


\section{Determinants of Adolescent Physical Activity and Inactivity Patterns \\ Penny Gordon-Larsen, Robert G. McMurray and Barry M. Popkin \\ Pediatrics 2000; $105 ; \mathrm{e} 83$ \\ DOI: $10.1542 /$ peds.105.6.e83}

\section{Updated Information \&}

Services

\section{References}

Subspecialty Collections

Permissions \& Licensing

Reprints including high resolution figures, can be found at:

http://pediatrics.aappublications.org/content/105/6/e83

This article cites 29 articles, 3 of which you can access for free at: http://pediatrics.aappublications.org/content/105/6/e83.full\#ref-list-1

This article, along with others on similar topics, appears in the following collection(s):

Adolescent Health/Medicine

http://classic.pediatrics.aappublications.org/cgi/collection/adolescent _health:medicine_sub

Information about reproducing this article in parts (figures, tables) or in its entirety can be found online at:

https://shop.aap.org/licensing-permissions/

Information about ordering reprints can be found online:

http://classic.pediatrics.aappublications.org/content/reprints

Pediatrics is the official journal of the American Academy of Pediatrics. A monthly publication, it has been published continuously since . Pediatrics is owned, published, and trademarked by the American Academy of Pediatrics, 141 Northwest Point Boulevard, Elk Grove Village, Illinois, 60007. Copyright @ 2000 by the American Academy of Pediatrics. All rights reserved. Print ISSN:

\section{American Academy of Pediatrics}






\title{
OFFICIAL JOURNAL OF THE AMERICAN ACADEMY OF PEDIATRICS
}

\author{
Determinants of Adolescent Physical Activity and Inactivity Patterns \\ Penny Gordon-Larsen, Robert G. McMurray and Barry M. Popkin \\ Pediatrics 2000;105; 83 \\ DOI: $10.1542 /$ peds.105.6.e83
}

The online version of this article, along with updated information and services, is located on the World Wide Web at:

http://pediatrics.aappublications.org/content/105/6/e83

Pediatrics is the official journal of the American Academy of Pediatrics. A monthly publication, it has been published continuously since . Pediatrics is owned, published, and trademarked by the American Academy of Pediatrics, 141 Northwest Point Boulevard, Elk Grove Village, Illinois, 60007. Copyright @ 2000 by the American Academy of Pediatrics. All rights reserved. Print ISSN:

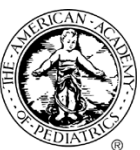

